\title{
Intensive Logopädie hilft bei chronischer Aphasie
}

Breitenstein $C$ et al. Intensive speech and language therapy in patients with chronic aphasia after stroke: a randomised, open-label, blinded-endpoint, controlled trial in a health-care setting. Lancet 2017; 389: 1528-1538

Jeder fünfte Patient ist nach einem Schlaganfall von einer chronischen Aphasie betroffen. Die Sprech- und Sprachfähigkeit bestimmt maßgeblich, ob Rehabilitationsziele erreicht werden. In der randomisierten FCET2EC-Studie verbesserte eine 3-wöchige Intensivtherapie die expressive Kommunikationskompetenz und die Lebensqualität von Betroffenen und ihren Partnern. Bei allen Patienten lag der Schlaganfall länger als 1 Jahr zurück.

Metaanalysen hatten gezeigt, dass bei einer chronischen Aphasie intensive logopädische Programme helfen. Die Aussagekraft der Untersuchungen war wegen 
kleiner Gruppengrößen, fehlender Kontrollgruppen und geringen Trainingsvolumina begrenzt. FCET2EC (From Controlled Experimental Trial 2 Everyday Communication) war eine Parallelgruppenstudie, an der 19 ambulante und stationäre Therapiezentren teilnahmen, die jährlich 699 Patienten (median) nach einem Schlaganfall betreuten. Die Studienleitung an der Universität Münster bestand aus Neurologen, Neurolinguisten, Neuropsychologen, 1 Biostatistiker und 1 Patienten. Neben der Aphasie waren Einschlusskriterien u. a.:

- Alter 18-70 Jahre

- ischämischer/hämorrhagischer Schlaganfall vor > 6 Monaten

- Deutsch als Erstsprache

- rudimentäres Sprechen und Verstehen

Die basale Kommunikationsfähigkeit wurde mit dem Aachener Aphasietest (AAT) überprüft. In der Interventionsgruppe erhielten 78 Patienten eine 3-wöchige Intensivtherapie mit mindestens 10 Wochenstunden Logopädie und 5 Wochenstunden Selbstübungen, die überwiegend am Computer erfolgten. In der Kontrollgruppe mit 78 Patienten fand das Programm mit 3-wöchiger Verzögerung statt. In beiden Gruppen bekamen 19 Patienten (Intervention) und 15 Patienten (Kontrollen) eine 5-wöchige Intensivintervention. Kriterium für einen Behandlungserfolg war eine Verbesserung im Amsterdam-Nijmegen-Everyday-Language-Test (ANELT), der vor und nach dem Programm sowie nach 6 Monaten erfolgte.

Die Gruppen waren hinsichtlich klinischer und soziodemografischer Daten insgesamt und insbesondere hinsichtlich der initialen Kommunikationsfähigkeit vergleichbar. In der Interventionsgruppe lag der Schlaganfall durchschnittlich 43 Monate und in der Kontrollgruppe 27 Monate zurück. Während des 3-Wochen-Kurses erfolgten 22,5 vs. 1,5 Stunden Sprachtherapie. Alle Patienten absolvierten das Programm vollständig. Verglichen mit den Kontrollen überzeugte das intensive Training mit signifikant besseren Ergebnissen:
- Verbesserung der expressiven Kommunikationsfähigkeit (ANELT-A; $p=0,0004$ )

- Bestätigung der Fortschritte in der verzögert behandelten Kontrollgruppe

- Wirkungssteigerung durch 5-WochenProgramm

- Effektstabilität nach 6 Monaten

Die Intervention beeinflusste die linguistischen Kompetenzen (SAP-Skala), die kommunikative Effektivität gegenüber dem Partner (CETI) und die Lebensqualität (SAQOL-39) positiv. Gruppenunterschiede bei der akustischen Verständlichkeit, körperlichen Behinderung und non verbalen kognitiven Funktionen bestanden nicht. Nur die Schwere des Schlaganfalls korrelierte mit dem Behandlungserfolg. Patienten mit einer weniger stark ausgeprägten Aphasie profitierten besonders.

FAZIT

In der 6-monatigen Nachbeobachtungsphase erhielten alle Patienten wöchentlich 1,5 Stunden Sprachtherapie. Daraus resultierte kein weiterer Fortschritt. Die Autoren sehen darin eine Bestätigung der aufwendigen und kostspieligen Intensivintervention. Die Verbesserungen im ANEL-Test seien zwar moderat ausgefallen, könnten aber die entscheidende Brücke für eine Wiedereingliederung sein. Die Studie untermauert die Relevanz der Sprachtherapie auch für Patienten mit einem länger zurückliegenden Schlaganfall.

Dr. med. Susanne Krome, Melle 